\title{
LA CÁTEDRA ISABEL MUÑOZ CARAVACA: UNA INICIATIVA PARA EL ESTUDIO Y EL FOMENTO DE LA IGUALDAD ENTRE HOMBRES Y MUJERES
}

\author{
CÁTEDRA ISABEL MUÑOZ CARAVACA: UMA INICIATIVA PARA O ESTUDO E \\ PROMOÇÃO DA IGUALDADE ENTRE HOMENS E MULHERES
}

\author{
ISABEL MUÑOZ CARAVACA DISCIPLINE: AN INITIATIVE FOR THE STUDY AND \\ PROMOTION OF EQUALITY BETWEEN MEN AND WOMEN
}

\author{
Nieves HERNÁNDEZ-ROMERO ${ }^{1}$ \\ Concepción CARRASCO CARPIO ${ }^{2}$ \\ Soledad ANDRÉS GÓMEZ ${ }^{3}$
}

RESUMEN: El pasado mes de junio de 2019 ha sido presentada la Cátedra de investigación Isabel Muñoz Caravaca, fruto de un convenio entre la Universidad de Alcalá y el Instituto de la Mujer de Castilla-La Mancha. El objetivo de esta cátedra es promover la investigación en temas relacionados con la violencia de género, la igualdad y la visibilización de la contribución de las mujeres a lo largo de la historia. Con este propósito se realizarán durante el primer año actividades de $\mathrm{I}+\mathrm{D}+\mathrm{I}$, así como la transferencia y divulgación de los resultados de investigaciones relacionadas con los estereotipos y prejuicios de género y su percepción y vivencia por adolescentes de la región.

Este trabajo expone la fundamentación que sustenta las primeras actividades de investigación y divulgación de dicha cátedra.

PALABRAS CLAVE: Género. Investigación. Igualdad. Transferencia de resultados.

RESUMO: A disciplina de pesquisa Isabel Muñoz Caravaca foi introduzida em junho de 2019 como resultado de um acordo entre a Universidade de Alcalá e o Instituto da Mulher de Castela-Mancha. O objetivo dessa disciplina é promover pesquisas sobre questões relacionadas à violência de gênero, igualdade e visibilidade da contribuição das mulheres ao longo da história. Com esse propósito, durante o primeiro ano serão realizadas atividades de PD\&I (Pesquisa, Desenvolvimento e Inovação) bem como a transferência e divulgação dos resultados de pesquisas relacionadas a estereótipos e preconceitos de gênero e sua percepção e experiência por adolescentes da região. Este trabalho expõe as bases que sustentam as primeiras atividades de pesquisa e divulgação da referida disciplina.

PALAVRAS-CHAVE: Gênero. Pesquisa. Igualdade. Transferência de resultados.

${ }^{1}$ Universidad de Alcalá (UAH), Alcalá de Henares - España. Profesora Contratada del Departamento de Ciencias de la Educación, Área de Música. ORCID: http://orcid.org/0000-0002-4371-157X. E-mail: nieves.hernandez@uah.es

${ }^{2}$ Universidad de Alcalá (UAH), Alcalá de Henares - España. Profesora Titular del Departamento de Economía, Área de Sociología. ORCID: https://orcid.org/0000-0002-5605-4468. E-mail: concha.carrasco@uah.es

${ }^{3}$ Universidad de Alcalá (UAH), Alcalá de Henares, España. Profesora Contratada Doctora, Departamento de Ciencias de la Educación, Área de Psicología Evolutiva y de la Educación. ORCID: https://orcid.org/0000-00031187-9308. E-mail: soledad.andres@uah.es 
ABSTRACT: The Isabel Muñoz Caravaca research discipline was introduced in June 2019 as a result of an agreement between the University of Alcala and the Castilla-Mancha Women's Institute. The purpose of this discipline is to promote research on issues related to genderbased violence, equality, and visibility of women's contributions throughout history. For this purpose, during the first year activities of RD\&I (Research, Development and Innovation) will be carried out, as well as the transfer and dissemination of the results of research related to gender stereotypes and prejudices and their perception and experience by adolescents in the region. This work exposes the bases that support the first research activities and dissemination of this discipline.

KEYWORDS: Gender. Research. Equality. Transfer of results.

\section{Origen}

La idea de esta cátedra de investigación surge a raíz de la promulgación de la Ley de Igualdad de Castilla-La Mancha (Ley 12/2010, de 18 de noviembre, de igualdad entre mujeres y hombres de Castilla-La Mancha), que en su artículo 16 establece la creación de un Centro de Estudios e Investigaciones de la Igualdad de Género.

1. El Instituto de la Mujer de Castilla-La Mancha impulsará la creación de un Centro de Estudios e Investigaciones de la Igualdad de Género.

2. El Centro deberá analizar y realizar estudios e investigaciones para hacer visibles las discriminaciones por razón de sexo, evaluará las políticas de igualdad y promoverá las estrategias y medidas para alcanzar la igualdad real entre mujeres y hombres (ESPAÑA, 2010).

Después de un largo proceso, en marzo de 2019 se firmó el convenio de colaboración entre el Instituto de la Mujer de Castilla-La Mancha y la Universidad de Alcalá para la creación de esta cátedra. El objetivo principal es la promoción de la igualdad entre mujeres y hombres y la erradicación de la violencia de género.

La presentación oficial se celebró el 21 de junio de 2019, en un acto presidido por D. José Vicente Saz, rector de la Universidad de Alcalá, y Da Araceli Martínez, directora en funciones del Instituto de la Mujer de Castilla-La Mancha, con la asistencia de distintas personalidades. 
Imagen 1 - Presentación de la Cátedra

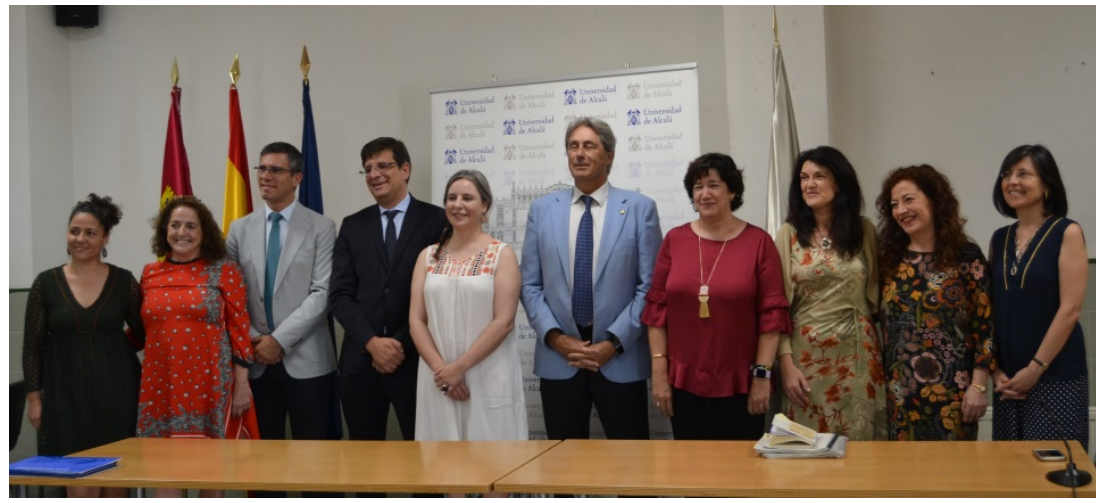

Fuente: Gobierno de Castilla-La Mancha (2019)

\section{Por qué Isabel Muñoz Caravaca}

Isabel Muñoz Caravaca (1848-1915), nacida en Madrid, obtuvo el título de Magisterio. Había estudiado música con Manuel de la Mata, maestro y secretario del Conservatorio de Madrid, y también francés, como era habitual en la época.

En 1874 contrajo matrimonio con Ambrosio Moya de la Torre, hombre de gran formación e ideas progresistas, catedrático de instituto de matemáticas, y después de universidad, ya viudo y que le doblaba la edad. De este matrimonio nacieron tres hijos.

Al enviudar en 1895, Isabel Muñoz Caravaca decidió ejercer como maestra, obteniendo en propiedad la plaza de la escuela de niñas de Atienza, donde vivió quince años. Al parecer pasó momentos difíciles, criticada en un entorno que no veía con buenos ojos su independencia y sus opiniones. En 1902 renunció a la plaza de maestra, aunque siguió viviendo en el pueblo hasta 1910, fecha en que se trasladó a Guadalajara. Desde ese momento y hasta su muerte publicó frecuentemente y bajo diferentes nombres en la prensa local. Esta constante presencia en la prensa y su creciente compromiso con la izquierda social provocó la enemistad de los sectores más conservadores de la capital.

Mientras fue maestra en Atienza también impartió clase en la Escuela nocturna para adultos, y preparaba a algunas jóvenes para acceder a la Escuela Normal. Escribió varios manuales para la instrucción: Principios de Aritmética (Madrid: Librería de Hernando, 1899) para la enseñanza primaria; Elementos de la Teoría del Solfeo (Madrid: Tipolitografía de R. Péant). Se dedicó especialmente a la astronomía, tema sobre el que publicó varios artículos, colaborando hacia 1905 con Camille Flammarion, famoso astrónomo, fundador de la Sociedad Astronómica Francesa, a la cual perteneció Isabel Muñoz Caravaca. 
Colaboró con los periódicos progresistas de Guadalajara, aunque la destrucción de muchos de ellos ha impedido que se pueda conocer la mayoría de sus escritos. Lejos de dedicarse a los escasos temas que eran permitidos a las pocas mujeres que publicaban en prensa, Isabel Muñoz Caravaca opinaba sobre todo tipo de cuestiones. Se mostró muy reivindicativa no solo en lo referente a la instrucción femenina, sino a la de toda la sociedad. Isabel Muñoz Caravaca defendía fervientemente la igualdad moral e intelectual de mujeres y hombres, y se declaraba feminista, partidaria del voto femenino y de la igualdad absoluta de derechos civiles y políticos.

No solo se preocupó de las mujeres; era defensora de los derechos de los oprimidos, defendiendo la mejora de las condiciones de los obreros; fue contraria a la pena de muerte. También fue defensora de los animales, emprendiendo campañas contra ritos y costumbres en los que eran maltratados. Publicó numerosos artículos en la prensa progresista de la provincia de Guadalajara. Uno de los títulos en los que participó con mayor asiduidad fue Flores y Abejas, vinculado a los círculos republicanos y socialistas, aunque en sus inicios se declaraba ajeno a la política y mantuvo una cierta línea intrascendente. También publicó en otros periódicos como La Alcarria Obrera, La Juventud Obrera o La Alcarria Ilustrada.

La defensa de sus ideas la enfrentó con frecuencia a los dirigentes más conservadores de la provincia y sufrió numerosos ataques (CALERO, 2006).

Sin duda fue una figura que merece ser recordada, y qué mejor homenaje que instituir esta cátedra de investigación con su nombre.

\section{Imagen 2 - Retrato de Isabel Muñoz Caravaca}

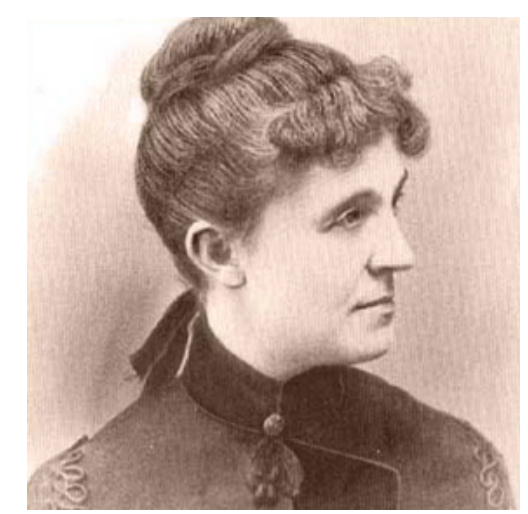

Fuente: Gobierno de Castilla-La Mancha

El logotipo de la cátedra quiere ser un homenaje a Isabel Muñoz Caravaca. En él se representa el perfil de la parte superior del cuerpo de una mujer anónima, como lo fueron tantas 
durante siglos. El color morado tiene un carácter reivindicativo. La firma es la original de Isabel Muñoz Caravaca.

Imagen 3 - Logotipo de la Cátedra de investigación Isabel Muñoz Caravaca

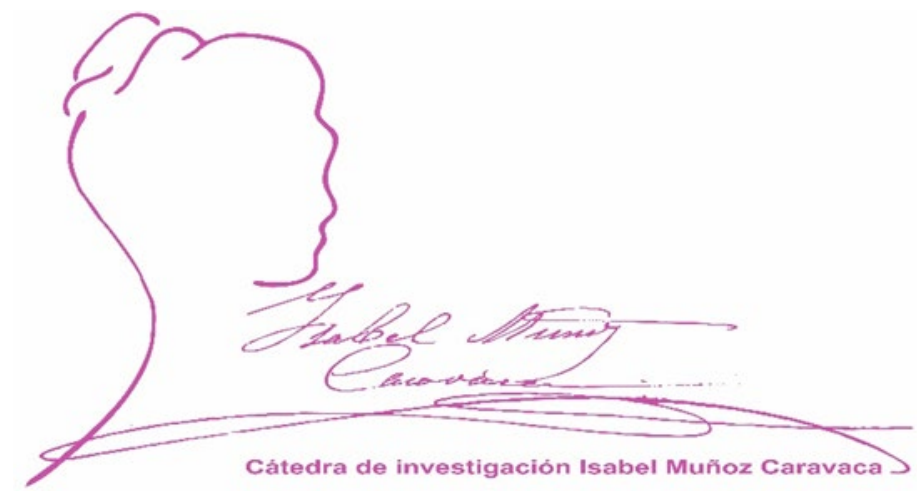

Fuente: Universidad de Alcalá (UAH) (2020)

\section{Quiénes somos}

El grupo que constituye esta cátedra está formado por profesorado investigador de la Universidad de Alcalá de diversas áreas de conocimiento, por alumnado de la misma institución y algunos investigadores externos a ella. Asimismo, la toma de decisiones sobre las actividades de la cátedra está gestionada por una comisión mixta compuesta por personal del Instituto de la Mujer de Castilla-La Mancha y de la UAH.

Son todas ellas personas relacionadas con los estudios de género a través de líneas de investigación muy variadas. Entre ellas se pueden citar la prevención de la mutilación genital femenina en Castilla-La Mancha, música y mujeres, transmisión de estereotipos de género en músicas populares urbanas, desarrollo psicosocial y género en la adolescencia, perspectiva de género en el análisis de las relaciones interpersonales y el clima escolar, análisis de textos escolares y lenguaje inclusivo, evaluación de servicios prestados por la Red de Puntos Municipales del Observatorio Regional de la Violencia de Género de la Comunidad de Madrid, análisis de materiales formativos para la sensibilización y prevención de la violencia de género, empoderamiento de mujeres inmigrantes de segunda generación a través de la educación, violencia de género en contextos de pobreza, cognición social y género, historia oral de las pioneras deportistas durante el franquismo en España, la construcción de la identidad de las deportistas durante la adolescencia y la adultez en contextos de ocio y de alto rendimiento, género y educación, violencia contra las mujeres, sexismo y amor romántico, etc. 


\section{Primer proyecto de investigación de la Cátedra}

El convenio se articula en varias fases. En la primera se desarrolla el proyecto "Percepción de la igualdad entre hombres y mujeres en la adolescencia de Castilla-La Mancha", entre marzo y noviembre de 2019.

El objetivo final es la promoción de la igualdad entre mujeres y hombres y la erradicación de la violencia de género. Con este fin, este proyecto pretende conocer las actitudes y percepciones de los y las adolescentes de Castilla-La Mancha acerca de cuestiones de igualdad entre mujeres y hombres. Se analizan así actitudes sexistas, mitos del amor romántico, estereotipos de género e influencia de las redes sociales.

En una primera fase se analizó la situación, se seleccionó bibliografía, se elaboró el informe jurídico sobre la violencia de género y un análisis del contexto español, se preparó el instrumento de recogida de información (cuestionario) y se seleccionó la muestra.

La selección de la muestra se realizó atendiendo a varios criterios. Tras valorar diversas cuestiones, se decidió realizar la encuesta al alumnado de $3^{\circ}$ y $4^{\circ}$ de la ESO en institutos públicos de Castilla-La Mancha.

Los cuestionarios se aplicaron la segunda quincena de septiembre de 2019. Posteriormente se procesarán los datos y se elaborará el informe correspondiente con los resultados.

\section{Fundamentación teórica}

El proyecto tiene su fundamento en la categoría de género, como construcción social, histórica y cultural. Los procesos de socialización de la infancia y la adolescencia están marcados por la diferenciación de género sentando las bases de la desigualdad de oportunidades en una sociedad basada en el sistema sexo-género. "No hay igualdad si la igualdad misma no es la base de la socialización" (VENEGAS, 2015, p. 68). La desigualdad a su vez supone discriminación y se vincula con las actitudes propias de la violencia de género, la cual se manifiesta de múltiples maneras. Es muy numerosa la producción científica en España que certifica las relaciones entre estas categorías y procesos (AMURRIO et al., 2008; DE MIGUEL, 2015; DÍAZ-AGUADO; CARVAJAL, 2011; DÍAZ-AGUADO; MARTÍNEZ; MARTÍNEZ, 2013; TORRES; ROBLES DE MARCO, 2014; SUBIRATS, 2010; VENEGAS, 2011).

Específicamente, una de las principales dimensiones a analizar es el sexismo. Este puede ser definido como una actitud hacia las personas en función de su pertenencia a grupos basados en el sexo biológico, sean hombres o mujeres. Toda evaluación que se haga de una persona 
atendiendo a la categoría sexual biológica puede ser etiquetada como sexista (EXPÓSITO; MOYA; GLICK, 1998). Para Rodríguez-Castro et al. (2010), el sexismo se produce ante cualquier evaluación que se realice sobre una persona en función de dicha categoría sexual biológica. Según Ferrer, Bosch, Ramis y Navarro (2006), el sexismo es una actitud negativa, una evaluación que solo incluye aspectos distorsionados de las mujeres, aunque sean encubiertos a partir de un tono positivo, aparentemente afectivo. Trabajos previos con muestras de estudiantes reportan que los hombres manifiestan más sexismo que las mujeres (RODRÍGUEZ-CASTRO; MAGALHAES, 2013). De Lemus, Castillo, Moya, Padilla y Ryan (2008) han señalado que es previsible que los hombres obtengan puntuaciones altas en el sexismo, ya que este contribuye a mantener el poder y la dominación sexual, de ahí que uno de los aspectos que se pretende analizar con este proyecto es la comparativa entre hombres y mujeres.

Por otra parte, la socialización es el proceso mediante el cual las personas aprenden las conductas y normas del entorno social (FERRER; BOSCH, 2013), por lo que se trata de un elemento clave en la reproducción de la desigualdad entre mujeres y hombres (LORENTE, 2007). En este proceso se conforma la identidad y se interiorizan las pautas culturales asignadas a cada género (MOSTEIRO; PORTO, 2017). En la socialización diferencial participan diferentes agentes educativos: familia, escuela, medios de comunicación, religiones, literatura, música, etc. En este sentido, el sistema educativo tiene un papel central en el fomento y desarrollo de una cultura igualitaria (RODRÍGUEZ MARTÍNEZ, 2011; AZORÍN, 2017; HEREDERO DE PEDRO, 2017), de ahí que la población objeto de estudio sea estudiantes de Educación Secundaria. En este sentido, el profesorado es el agente idóneo para reflexionar sobre la desigualdad de género y construir modelos de socialización alternativos que permitan la consecución de una igualdad real y efectiva.

Otro de los aspectos que se quiere incluir en la investigación es el que se relaciona con los mitos del amor romántico, definidos como el conjunto de creencias que se comparten socialmente sobre la supuesta "verdadera" naturaleza del amor (YELA, 2003). Estos representan el imaginario social sobre el significado que se le da al amor. Diversos estudios realizados en España confirman la vigencia del discurso romántico tanto en población general (BOSCH et al., 2008), como en población adolescente (RODRÍGUEZ-CASTRO et al., 2013).

Siendo la población objeto del presente estudio el alumnado de Educación Secundaria, adquiere especial relevancia el análisis de la calidad de las relaciones en el contexto de los encuentros cara a cara, así como en las redes sociales. El uso de internet y dispositivos móviles por niños y niñas y jóvenes en España viene estudiándose recientemente en el campo de las 
ciencias sociales. A día de hoy se cuenta con datos derivados de investigaciones de carácter longitudinal de distinto ámbito que aportan información relevante acerca del funcionamiento de niños y niñas, y jóvenes adolescentes, en los entornos virtuales de interacción (por ejemplo Garmendia, Jiménez, Casado, y Mascheroni, 2016; Instituto Nacional de Estadística, 2014).

Los cambios en las formas de comunicación y relación que han experimentado todas las personas, pero especialmente las más jóvenes, con el uso cotidiano de los espacios virtuales son hechos admitidos. De manera particular, las parejas adolescentes, ocupadas en las tareas evolutivas propias de la etapa -búsqueda de identidad, autonomía, inicio de las relaciones-, exploran nuevas vinculaciones afectivas con otra $u$ otras personas combinando las interacciones virtuales con las presenciales e influyendo de manera notable en los primeros aprendizajes del amor y de las relaciones de pareja (SÁNCHEZ-JIMÉNEZ et al., 2017) Así, investigaciones como las realizadas por Subrahmanyam y Smahel (2011) proponen modelos de análisis que parten de la idea de que el mundo en línea y fuera de línea están íntimamente conectados, influyendo en el desarrollo de la intimidad, los patrones de cortejo (ahora denominado "cibercortejo"), el compromiso de pareja, etc. en estas etapas tempranas de la vida. Y en sentido negativo, también pueden aumentar o promover sentimientos y conductas dañinas como los celos o el ciberacoso (DRAUCKER; MARTSOLF, 2010; ZWEIG; DANK; LACHMAN; YAHNER, 2013).

Con el fin de aportar conocimiento a un campo emergente, motivo asimismo de preocupación social, se analizan los recientes estudios desarrollados por equipos de investigación del campo de las ciencias sociales, prestando especial atención a la influencia de la variable de género para analizar las consecuencias de las conductas de abuso y victimización en las citas y relaciones de parejas adolescentes (SÁNCHEZ-JIMÉNEZ et al., 2018; REED et al., 2017; SÁNCHEZ-JIMÉNEZ et al., 2015).

Por lo tanto, a partir de la revisión de estudios previos con este sector de población joven, el objetivo es analizar este tipo de cogniciones y conductas en una muestra representativa de adolescentes de Castilla la Mancha. El análisis de esta población podría contribuir a que diferentes agentes de socialización, responsables en la educación de los y las adolescentes, tengan conocimiento del estado de la cuestión en esta región donde podría haber diferencias según el sexo, entre contextos rurales y urbanos, así como respecto a otras variables que dificulten una igualdad efectiva.

En síntesis, en el estudio de las relaciones entre adolescentes en los contextos cara a cara y virtuales, los investigadores e investigadoras han analizado cuatro categorías principalmente: género, sexualidad, amor-afectividad y cuerpo. Y los temas principales, de los que asimismo 
nos ocuparemos en el estudio que proyectamos, son: el sexismo, los estereotipos de género, los mitos del amor romántico y la influencia de las redes sociales como espacio para la violencia de género.

\section{Consideraciones finales}

Esta cátedra de investigación nace con el objetivo de investigar en temas relacionados con la igualdad de mujeres y hombres. Existen muchas formas de luchar contra las desigualdades de género, pero una fundamental es a través de la deconstrucción de los estereotipos generados en los procesos de socialización. Esto no es posible si no se analizan en profundidad los fundamentos, tanto teóricos como empíricos de la desigualdad. Una sociedad democrática debe ser igualitaria y si no lo es la democracia pierde fuerza y credibilidad.

La investigación junto con la transferencia de los conocimientos y resultados que se vayan produciendo, además de la recuperación de los nombres (y acciones) de mujeres anónimas, quieren ser los frutos de esta cátedra.

\section{REFERENCIAS}

AMURRIO, M. et al. Violencia de género en las relaciones de pareja adolescentes y jóvenes de Bilbao. Bilbao: Ayuntamiento de Bilbao y Universidad del País Vasco, 2008.

AZORÍN, C. M. Actitudes hacia la igualdad de género en una muestra de estudiantes de Murcia. Revista Complutense de Educación, v. 28, n. 1, p. 45-60, 2017.

BOSCH, E.; FERRER, M. V.; GARCÍA, M. E.; RAMIS, M. C.; MAS, M. C.; NAVARRO, C.; TORRENS, G. Del mito del amor romántico a la violencia contra las mujeres en la pareja. Madrid: Instituto de la Mujer, 2007. Disponible en:

http://centreantigona.uab.cat/izquierda/amor\%20romantico\%20Esperanza\%20Bosch.pdf. Acceso en: 1 jun. 2019.

CALERO, J. J. Isabel Muñoz Caravaca (1848-1915). Mujer de un siglo que no ha llegado aún. Ciudad Real: Almud Ediciones, 2006.

DE MIGUEL, V. Percepción de la violencia de género en la adolescencia y la juventud. Madrid: Ministerio de Sanidad, Política Social e Igualdad, 2015.

DRAUCKER, C. B.; MARTSOLF, D. S. The role of electronic communication technology in adolescent dating violence. Journal of Child and Adolescent Psychiatric Nursing, v. 23, n. 3, p. 133-142, 2010. DOI: https://doi.org/10.1111/j.1744-6171.2010.00235.x

ESPAÑA. Ley 12/2010, de 18 de noviembre. Igualdad entre mujeres y hombres de CastillaLa Mancha, 2010.

RIAEE - Revista Ibero-Americana de Estudos em Educação, Araraquara, v. 15, n. esp. 4, p. 2687-2698, dez., 2020. e-ISSN: 1982-5587 
EXPÓSITO, F.; MOYA, M.; GLICK, P. Sexismo ambivalente: medición y correlatos. Revista de Psicología Social, v. 13, n. 2, p. 159-169, 1998.

FERRER, V.; BOSCH, E. Del amor romántico a la violencia de género: Para una coeducación emocional en la agenda educativa. Profesorado: Revista de currículum y formación del profesorado, v. 17, n. 1, p. 107-122, 2013.

FERRER, V.; BOSCH, E.; RAMIS, M. C.; NAVARRO, C. Las creencias y las actitudes sobre la violencia contra las mujeres en la pareja: Determinantes sociodemográficos, familiares y formativos. Anales de Psicología, v. 22, n. 2, p. 251-259, 2006.

GARMENDIA, M.; JIMÉNEZ, E.; CASADO, M. A.; MASCHERONI, G. Net children go mobile: riesgos y oportunidades en internet y el uso de dispositivos móviles entre menores españoles (2010-2015). Madrid: Red.es/Universidad del País Vasco/Euskal Herriko Unibertsitatea, 2016.

GOBIERNO DE CASTILLA-LA MANCHA. EI Gobierno regional y la Universidad de Alcalá ponen en marcha la Cátedra de investigación de género 'Isabel Muñoz Caravaca'. 21 jun. 2019. Disponible en: https://www.castillalamancha.es/node/290739. Acceso en: 21 jun. 2019.

GOBIERNO DE CASTILLA-LA MANCHA. Enciclopedia de mujeres “Olivia Sabuco". Disponible en: https://institutomujer.castillalamancha.es/centro-de-documentacion-ybiblioteca-luisa-sigea/enciclopedia-de-mujeres-oliva-sabuco/mujeres/guadalajara. Acceso en: 25 jun. 2019.

HEREDERO DE PEDRO, C. Género y coeducación. Madrid: CCOO Enseñanza, 2017.

INSTITUTO NACIONAL DE ESTADÍSTICA. España en cifras 2014. Madrid, España: Autor, 2014.

LEMUS, S.; CASTILLO, M.; MOYA, M.; PADILLA, J. L.; RYAN, E. Elaboración y validación del Inventario de Sexismo Ambivalente para adolescentes. International Journal of Clinical and Health Psychology, v. 8, n. 2, p. 537-562, 2008.

LORENTE, M. Violencia de género, educación y socialización: acciones y reacciones. Revista de Educación, n. 342, p. 19-35, 2007.

MOSTEIRO, M. J.; PORTO, A. M. Análisis de los estereotipos de género en alumnado de formación profesional: diferencias según sexo, edad y grado. Revista de Investigación Educativa, v. 35, n. 1, p. 151-165, 2017.

REED, L.; TOLMANB, R.; WARDC, M. Gender matters: Experiences and consequences of digitaldating abuse victimization in adolescent dating relationships. Journal of Adolescence, v. 59, p. 79-89, 2017. DOI: https://doi.org/10.1016/j.adolescence.2017.05.015

RODRÍGUEZ CASTRO, Y.; MAGALHAES, M. J. El sexismo moderno en estudiantes universitarios/as portugueses/as. Revista Interdisciplinar de Ciencias Sociales y Humanas, v. 1, n. 2, p. 113-121, 2013. 
RODRÍGUEZ MARTÍNEZ, C. Género y cultura escolar. Madrid: Morata, 2011.

RODRÍGUEZ-CASTRO, Y. et al. La fiabilidad y validez de la escala de mitos hacia el amor: las creencias de los y las adolescentes. Revista de Psicología Social, v. 28, n. 2, p. 157-168, 2013.

SÁNCHEZ JIMÉNEZ, V.; MUÑOZ-FERNÁNDEZ, N.; LUCIO LÓPEZ, L. A.; ORTEGARUÍZ, R. Ciberagresión en parejas adolescentes: Un estudio transcultural España-México.

Revista Mexicana de Psicología, v. 34, n. 1, p. 46-54, 2017.

SÁNCHEZ JIMÉNEZ, V.; MUÑOZ-FERNÁNDEZ, N.; ORTEGA-RUIZ, R. "Cyberdating Q_A": An instrument to assess the quality of adolescent dating relationships in social networks. Computer in Human Science, v. 48, p. 78-86, 2015.

SÁNCHEZ-JIMÉNEZ, V.; MUÑOZ-FERNÁNDEZ, N.; ORTEGA-RIVERA, J. Efficacy evaluation of "Dat-e Adolescence": A dating violence prevention program in Spain. PLoS ONE v. 13, n. 10, 2018. DOI: https://doi.org/10.1371/journal.pone.0205802

SUBIRATS, M. ¿Coeducación o escuela segregada? Un viejo y persistente debate. Revista de la Asociación de Sociología de la Educación (RASE), v. 3, n. 1, v. 143-158, 2010.

TORRES, C.; ROBLES, J.M; DE MARCO, S. El ciberacoso como forma de ejercer la violencia de género en la juventud: un riesgo en la sociedad de la información y el conocimiento. Madrid: Ministerio de Sanidad, Política Social e Igualdad, 2014.

VENEGAS, M. Un modelo sociológico para investigar las relaciones afectivo-sexuales. Revista Mexicana de Sociología, v. 73, n. 4, p. 559-589, 2011.

YELA, C. La otra cara del amor: Mitos, paradojas y problemas. Encuentros en Psicología Social, v. 1, n. 2, p. 263-267, 2003.

ZWEIG, J. M.; DANK, M.; LACHMAN, P.; YAHNER, J. Technology, teen dating violence and abuse, and bullying. Final report. Washington, DC, E.U.: Urban Institute, 2013. Disponible en: http://www.urban.org/research/publication/technology-teen-dating-violencea abuse-and-bullying. Acceso en: 21 jun. 2019. 


\section{Cómo referenciar este artículo}

HERNÁNDEZ-ROMERO, N.; CARRASCO CARPIO, C.; ANDRÉS GÓMEZ, S. La cátedra Isabel Muñoz Caravaca: una iniciativa para el estudio y el fomento de la igualdad entre hombres y mujeres. Revista Ibero-Americana de Estudos em Educação, Araraquara, v. 15, n. esp. 4, p. 2687-2698, dez., 2020. e-ISSN: 1982-5587. DOI: https://doi.org/10.21723/riaee.v15iesp4.14515

Remitido el: 10/09/2019

Revisiones requeridas el: 10/01/2020

Aprobado el: 30/04/2020

Publicado el: 01/12/2020 